\title{
STEREO Observations of Energetic Ions in Corotating Interaction Regions During the May 2007 Solar Events
}

\author{
R. Bučík · U. Mall · R. Gómez-Herrero • A. Korth • \\ G.M. Mason
}

Received: 8 December 2008 / Accepted: 12 July 2009 / Published online: 3 September 2009

(C) The Author(s) 2009. This article is published with open access at Springerlink.com

\begin{abstract}
Elemental composition and energy spectra of $\sim 0.1-1.0 \mathrm{MeV} / \mathrm{n}$ heavy ions were analyzed in two corotating interaction region (CIR) events starting on 18 May (CIR1), and 24 May (CIR2), 2007, that occurred during two weak solar energetic particle (SEP) events. The data were taken by the Suprathermal Ion Telescope (SIT) instruments onboard STEREO-A and B. The ion intensity increases related to the CIR2 were much more pronounced compared to those associated with the CIR1, although the total pressure, the magnetic field magnitude and the speed difference between high and slow solar wind were less enhanced. We found that the ratio of CIR2 to CIR1 helium fluence decreases with energy as a power-law below $\sim 1 \mathrm{MeV} / \mathrm{n}$. The energy spectra of hydrogen and helium inside the CIR2 region are much softer compared to the spectra observed in the CIR1 region. Based on these findings, we argue that the larger intensities in the CIR2 event could be due to an additional source population. The elemental abundances in CIR2 and the high-speed stream of the CIR1 region have a SEP-like composition. The energy spectra of hydrogen and helium inside the CIR2 were close to the preceding SEP event spectra. The similarity in the elemental abundances and in the spectral slopes suggests that the seed population of CIR2 are ions from the preceding SEP event. For the CIR1 event the ions seem to be contaminated by SEPs.
\end{abstract}

Keywords Energetic particles, abundances $\cdot$ Flares, energetic particles

STEREO Science Results at Solar Minimum

Guest Editors: Eric R. Christian, Michael L. Kaiser, Therese A. Kucera, O.C. St. Cyr

R. Bučík $(\varangle) \cdot$ U. Mall · A. Korth

Max-Planck-Institut für Sonnensystemforschung, 37191 Katlenburg-Lindau, Germany

e-mail: bucik@mps.mpg.de

R. Bučík

Institute of Experimental Physics, Slovak Academy of Sciences, 04001 Košice, Slovakia

R. Gómez-Herrero

Universität Kiel, 24118 Kiel, Germany

G.M. Mason

Applied Physics Laboratory, Johns Hopkins University, Laurel, MD 20723, USA 


\section{Introduction}

The interaction of solar wind high-speed streams (HSSs), emerging from solar coronal holes, with the preceding slow-speed solar wind streams leads to the formation of compression regions called corotating interaction regions (CIRs). Often a pair of forward and reverse shocks is formed after the interaction of the two streams (e.g., Hundhausen and Gosling, 1976). These shocks can accelerate particles up to a few $\mathrm{MeV} / \mathrm{n}$ in energy. In particular, energetic ions observed in HSSs at 1 AU are expected to be energized at the CIR reverse shocks and propagate back toward the Sun along the magnetic field (Barnes and Simpson, 1976; Sanderson et al., 1995). The energetic ions from CIRs are also known to have very characteristic element abundances that distinguish them from other heliospheric energetic particle populations. The average heavy-ion composition of CIR elemental abundances is very close to the average fast solar wind composition (e.g., Mason et al., 2008a). It is known that shock acceleration models require energies well above the solar wind speed. Particles are thought to be accelerated either from the suprathermal tail of the solar wind distribution (Fisk and Lee, 1980) or the thermal solar wind ions are thought to be pre-accelerated through a statistical process inside the CIR to reach speeds above the shock acceleration threshold (Richardson, 1985; Schwadron, Fisk, and Gloeckler, 1996).

Early observations of CIR-associated particle events led to the idea that ions are not accelerated out of low energy solar energetic particle (SEP) source population (Christon and Simpson, 1979; Richardson et al., 1993). However, more recent observations (Sanderson et al., 1995; Torsti, Anttila, and Sahla, 1999; Malandraki et al., 2007, 2008), and statistical (Desai et al., 1998; Mason et al., 2008a) studies of corotating energetic particle phenomena suggest that sub-MeV particles from SEP events may provide a seed population for CIR acceleration. Such a sub-MeV particle population appears to be an attractive source since it can supply ions with energies above the threshold energy which is required for the ion acceleration by the CIR shocks.

The Ulysses energetic ion observations over the south pole revealed intensity increases of the recurrent $1.8-3.8 \mathrm{MeV}$ proton intensity peak after the transient events, which led Sanderson et al. (1995) to suggest that the energetic solar particles from each large solar event provide the seed particles for the recurrent CIRs to accelerate. In a single CIR event Torsti, Anttila, and Sahla (1999) observed a hardening of the energy spectra and intensity increases even above $20 \mathrm{MeV}$ in the late-phase of the event, not seen around the major peak at lower energies. They proposed a re-acceleration of SEPs, injected two days earlier, by the CIR. Malandraki et al. $(2007,2008)$ examined elemental composition of the energetic particle fluxes recorded at Ulysses at 5 AU during the periods of SEP/CIR combinations. They found the $\mathrm{C} / \mathrm{O}$ ratio consistent with SEP values and suggest as one possibility that the CIR reverse shocks re-accelerated particles of this species from the SEP events themselves. A survey of CIR events over the past solar cycle has revealed ${ }^{3} \mathrm{He}$ abundance several times higher than in the solar wind, showing that remnant impulsive flare suprathermal ions are accelerated in these events (Mason et al., 2008a). The same study shows no clear solar cycle dependence of He peak intensities. Similarly, a lack of solar cycle dependence of the proton peak intensity has been found in a survey of CIR events observed between 1978 and 1986 (Richardson et al., 1993). This observation led Richardson et al. (1993) to the suggestion that ambient low-energy solar particles, which are expected to be more abundant around solar maximum, do not provide a significant seed population for the acceleration by corotating shocks. However, they reported SEP-like corotating ion abundances at solar maximum and mentioned an SEP contamination or the acceleration of low-energy SEPs by corotating shocks. In analyzing the measurements associated with all compression regions 
observed by Ulysses, Desai et al. (1998) concluded that the correlation between the proton intensity and the magnetic compression ratio was not observed during periods of high solar activity, because of significant variations in the seed intensity that occurred due to sporadic contributions from transients. They have drawn an analogy with observations which have shown that sub-MeV/n particles from transient solar events are an important constituent of the seed population that is available for an acceleration at interplanetary shocks (e.g., Tan et al., 1989).

Mewaldt et al. (2008b) reported the observation of two small SEP events detected by instruments on ACE and STEREO-A and B in May of 2007. Both events were associated with small flares and relatively fast coronal mass ejections (CMEs). The first event started on 19 May 2007, the second on 23 May 2007. These two low-intensity SEP events are of special interest because these events occurred between the onsets of two CIR events on 18 and 24 May. The events, observed around solar minimum, present an ideal opportunity to investigate whether SEPs can be the source population for CIRs. Roelof et al. (1992) pointed out that during sustained high solar activity the inner heliosphere acts as a reservoir for low energy ions from transient events. Therefore, observing the two above mentioned CIR events during solar minimum, a time period during which transient events are rare, reduces the likelihood that the measured CIR abundances are contaminated by SEPs.

We used the above mentioned time period in this study to survey solar particle and CIR associated events and to examine the temporal variations of particle fluxes, energy spectra, and to derive elemental abundances of the SEP and CIR populations.

\section{Observations}

The measurements presented here were made with the Suprathermal Ion Telescope (SIT) (Mason et al., 2008b) onboard the STEREO spacecraft, A and B, launched in October 2006. In May 2007 STEREO-A, was at $0.96 \mathrm{AU}$, and STEREO-B, at 1.06 AU. The S/C were located at heliocentric longitudes of W06 and E03, respectively, near the ecliptic plane. The SIT instrument is a time-of-flight mass spectrometer which measures $\mathrm{H}$ to $\mathrm{Fe}$ ions from $20 \mathrm{keV} / \mathrm{n}$ to several $\mathrm{MeV} / \mathrm{n}$. The instrument has a geometric factor of $0.29 \mathrm{~cm}^{2} \mathrm{sr}$. In our study we also make use of energetic hydrogen ion measurements, made by the LET instrument (Mewaldt et al., 2008a), solar wind measurements made by the PLASTIC instrument (Galvin et al., 2008) and magnetic field measurements obtained by the magnetometer on STEREO (Acuña et al., 2008).

We begin by showing the May 2007 events in a broader context. We display in Figure 1 the energy spectrograms of all ions measured with SIT B during successive Carrington rotations (CRs). The lower panel shows the data between 17 May and 28 May. The upper panel shows the data in the corresponding part of the previous CR. It is not a priori clear that the enhancements with onsets on 18 May (day 138) and 24 May (day 144) 2007 are recurrent events. Figure 1 suggests that the event on 24 May was a recurrent one, since a faint increase in the number of Pulse-Height-Analysis (PHA) counts was observed during the previous CR around day 117. For the event observed on 18 May the situation is less clear, since only a weak ion enhancement was observed in the previous CR on day 113, and this enhancement occurred $\sim 2$ days later than we would have expected based on the solar rotation period.

\subsection{CIR Events}

Figure 2 shows He fluxes measured by the SIT instruments on STEREO for the two CIR/high-speed stream-associated ion events at the end of May 2007 together with the solar 


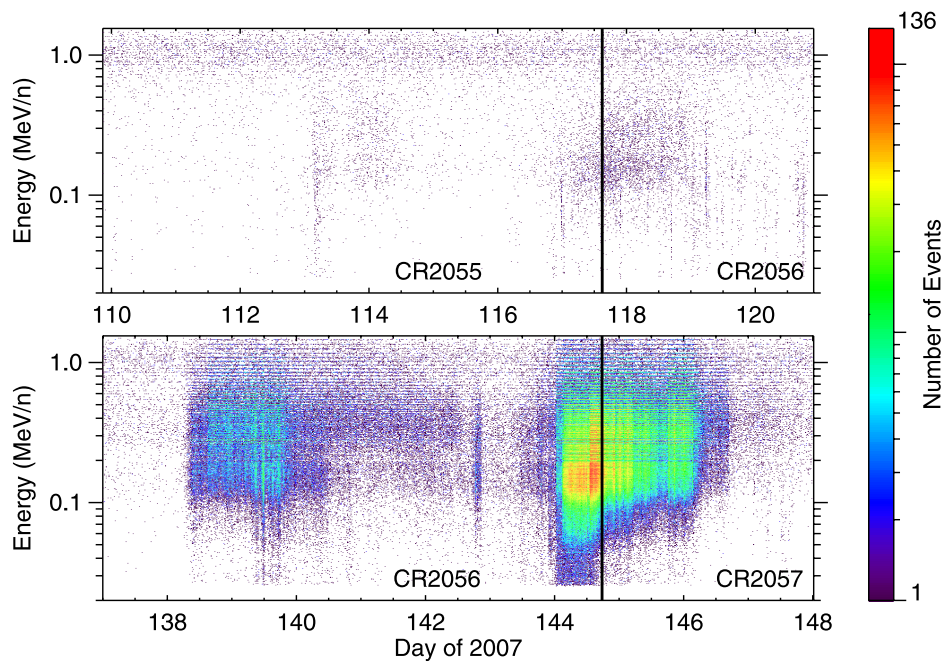

Figure 1 Kinetic energy $v s$. time diagrams for all ions observed with SIT for the successive Carrington rotations (CRs). The vertical black lines denote the start times of CR 2056 (upper panel) and CR 2057 (lower panel). Shown are the number of PHA ion events in SIT on STEREO-B.

wind plasma and magnetic field parameters measured by the PLASTIC instrument and the magnetometer sensor. Both of those events are included in the survey of Mason et al. (2009).

The top panels present hourly averaged values of the 0.16 to $0.91 \mathrm{MeV} / \mathrm{n} \mathrm{He}$ intensities, with the associated statistical errors. The lower panels show 10-min averages of the total pressure $P$, the magnetic field magnitude $|B|$, and the solar wind speed $V_{\mathrm{p}}$. The total pressure $P$ is given by the sum of the plasma and magnetic field pressure, i.e., $P=2 n_{\mathrm{p}} k T_{\mathrm{p}}+B^{2} / 2 \mu_{0}$, where $n_{\mathrm{p}}$ and $T_{\mathrm{p}}$ are the proton density and temperature, respectively, and $B$ is the magnetic field magnitude. Pairs of vertical dashed lines mark CIR leading and trailing edges, bounding the region of enhanced plasma densities and magnetic field intensities that precede the HSS and define the CIR itself (e.g., Richardson et al., 1993). The edges of the 17 May CIR (hereafter CIR1) were adopted from the 'List of Stream Interaction Regions' ${ }^{1}$ where CIRs were selected on the basis of plasma and field data. The whole time under investigation is complex since the interplanetary medium in the vicinity of the STEREO spacecraft on 22-23 May 2007 was influenced by the presence of an interplanetary CME (ICME) and another CIR (hereafter CIR2). The transit time of the ICME, reported in the 'List of ICMEs' (Level 3 data), is indicated by a gray shaded region in Figure 2. Liu et al. (2008) and Kilpua et al. (2009) have investigated the characteristics of the associated magnetic cloud. Kilpua et al. (2009) argued that a magnetic cloud observed by STEREO-B was associated with a partial halo CME launched on 19 May, whereas the magnetic cloud observed on STEREO-A was associated with another CME launched less than a day later. While the timing of the boundary of CIR 1 could be taken from the literature, the timing of the boundary of CIR2 had to be determined by us. The presence of the ICME around the leading edge of the HSS made the determination of the leading boundary of the CIR2 on STEREO-A less straightforward.

The leading edge of the interaction region is characterized by an increase in plasma density, temperature and magnetic field intensity and the trailing edge by decrease in these

${ }^{1}$ Level 3 data (http://www-ssc.igpp.ucla.edu/forms/stereo/). 


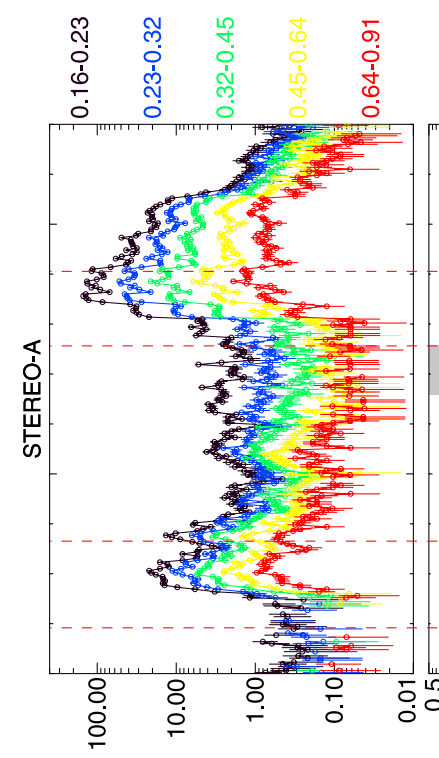

( $\_$U $\wedge$ əW ds $\left.\mathrm{s}_{2} \mathrm{mo}\right) / \partial \mathrm{H}$

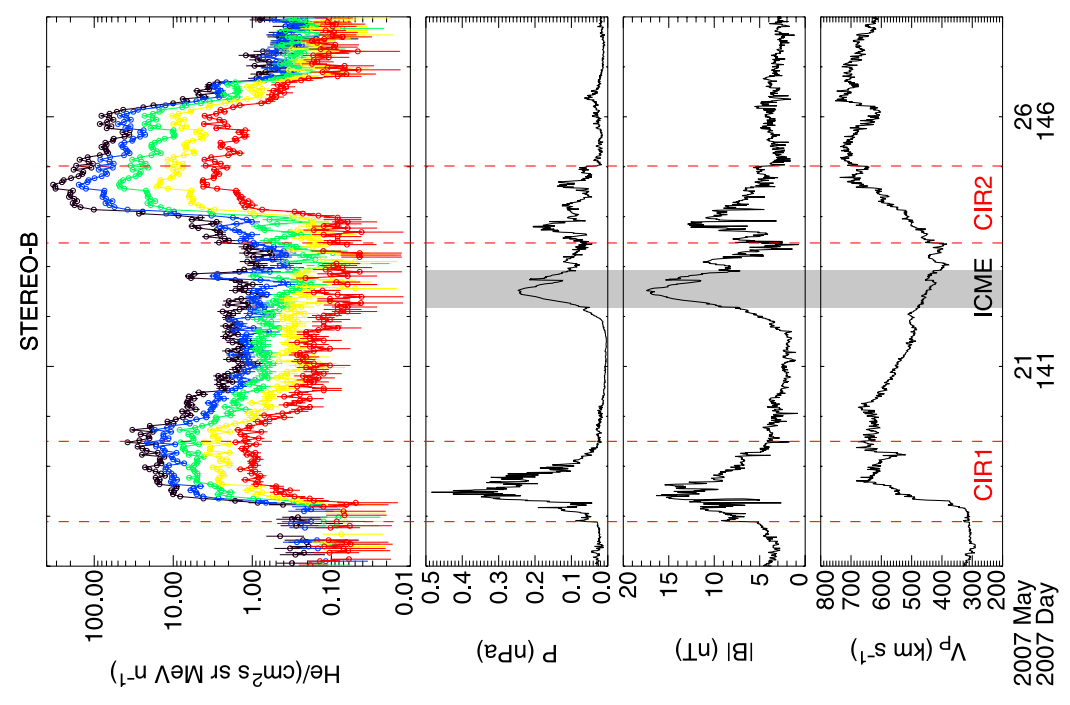

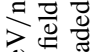

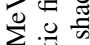

ब․

品

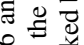

$\div$ 的

ธี

है

造

此

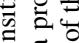

苞 零

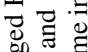
吗

过

급

어 냉 응

$\exists$

馬.

ㄴ.

के 을

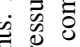

ฮั

वे न

응

을 34 氙 3 西 프 을 वे है 응 可 ए

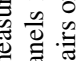
a 동ㅎㅇ 튀 을

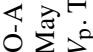
주요 I 10 政要

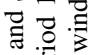
흘 os $\infty \neq$ 웡 牙 舀的市 的定兵

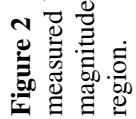


quantities (Smith and Wolfe, 1979). The solar wind speed increases at both edges. As far as there is no shock formed at these boundaries, the edges of the CIR represent continuous transition zones (Belcher and Davis, 1971) and there is some uncertainty in the location of the boundaries (Richardson and Zwickl, 1984). We inspect visually the time profile of the plasma density, temperature, magnetic field and solar wind speed and select the edges of the CIR2 at times when the characteristic variations in plasma parameters and magnetic field approximately coincide. Since the front and rear of the interaction region are also characterized by an abrupt increase and decrease in pressure (Burlaga, 1995), this parameter instead of $n_{\mathrm{p}}$ and $T_{\mathrm{p}}$ is shown in Figure 2.

The panels in Figure 2 displaying the solar wind speed show that the solar wind changes its speed from $\sim 300 \mathrm{~km} \mathrm{~s}^{-1}$ to $\sim 670 \mathrm{~km} \mathrm{~s}^{-1}$ in CIR1. The CIR2 HSS wind speeds of $\sim 710 \mathrm{~km} \mathrm{~s}^{-1}$ and $660 \mathrm{~km} \mathrm{~s}^{-1}$ are sampled by STEREO-B and A, respectively. CIR1 HSS and CIR2 HSS were separated from each other by less than a quarter of a solar rotation. In the case of CIR2, the HSS interacted with slow solar wind of higher speed compared to the CIR1 region. STEREO-B measured $>400 \mathrm{~km} \mathrm{~s}^{-1}$ and STEREO-A $\sim 470 \mathrm{~km} \mathrm{~s}^{-1}$ for the slow solar wind speed in the CIR2 region.

Our observation shows two CIR events with HSSs which are closely located in heliolongitude. Our measurements reveal in this case high ion fluxes in the CIR2 event. This finding is different from Richardson et al. (1993) who reported near quiet time ion fluxes in CIRs for closely spaced HSSs in heliolongitude. They argued that a sufficient high speed difference between the slow and fast interacting solar wind streams is needed for shocks to develop that can accelerate the ions to higher energies. However, they found no clear correlation between the CIR peak ion intensity and the speed difference between the high-speed solar wind and the preceding slow solar wind. Kobayashi et al. (2000) indeed showed a strong correlation among these parameters during solar minimum. Figure 2 shows clearly that the $\mathrm{He}$ intensity increase associated with the compression region CIR2 was higher than the He intensity increase associated with the compression region CIR1. This finding does not follow the correlation found by Kobayashi et al. (2000). In the energy band $0.32-0.45 \mathrm{MeV} / \mathrm{n}$, STEREO-A (B) measurements revealed in the CIR1 and CIR2 events a ${ }^{4} \mathrm{He}$ peak intensity (particles $/ \mathrm{cm}^{2} \mathrm{~s} \mathrm{sr} \mathrm{MeV/n}$ ) of 5.5 (8.3) and 21.0 (48.6), respectively. Other parameters like the magnetic field and the total pressure were greatly enhanced in CIR1 compared to the values found in CIR2. The peak pressure in CIR1 was 3.1 (2.4) times the value found in CIR2 at STEREO-A (B).

In order to quantitatively describe the ion intensities in the two CIRs, we compute the He fluences (particles $/ \mathrm{cm}^{2} \mathrm{sr} \mathrm{MeV/n}$ ). In Figure 3 we display the ratio of CIR2 to CIR1 He fluence in different energy ranges. The fluence ratios were computed for the pure CIR time periods (circles) and for the extended time periods which covers CIR and also the HSS region (squares). Figure 3 shows that the He intensity in CIR2 exceeded that in CIR1. At lower energies the intensity difference is an order of magnitude. Below $\sim 1 \mathrm{MeV} / \mathrm{n}$ the relative intensity of He ions decreases with energy, roughly linearly in $\log -\log$ scale (with approximate slope around -1 ).

\subsection{SEP Events}

The small SEP events of 19 May and 23 May had no obvious signature below $\sim 1 \mathrm{MeV} / \mathrm{n}$. Therefore, we use data from the LET instrument which covers a higher energy range (from $\sim 2 \mathrm{MeV} / \mathrm{n}$ to $\sim 30 \mathrm{MeV} / \mathrm{n}$ ) compared to the SIT instrument. Figure 4 shows STEREOA hourly averages of the $320 \mathrm{keV}$ to $10 \mathrm{MeV}$ proton intensities measured by SIT and LET. The solar wind speed is shown in the bottom panel of the figure and the compression regions CIR1 and CIR2 are marked. 
Figure 3 CIR2 to CIR1 He fluence

(particles $/ \mathrm{cm}^{2} \mathrm{sr} \mathrm{MeV} / \mathrm{n}$ ) ratio as a function of energy for STEREO-A (black symbols) and STEREO-B (red symbols). The ratios of fluences integrated inside the compression regions are indicated by circles and those taken throughout the flux enhancement, both inside the by squares. CIRs and in the HSSs are marked
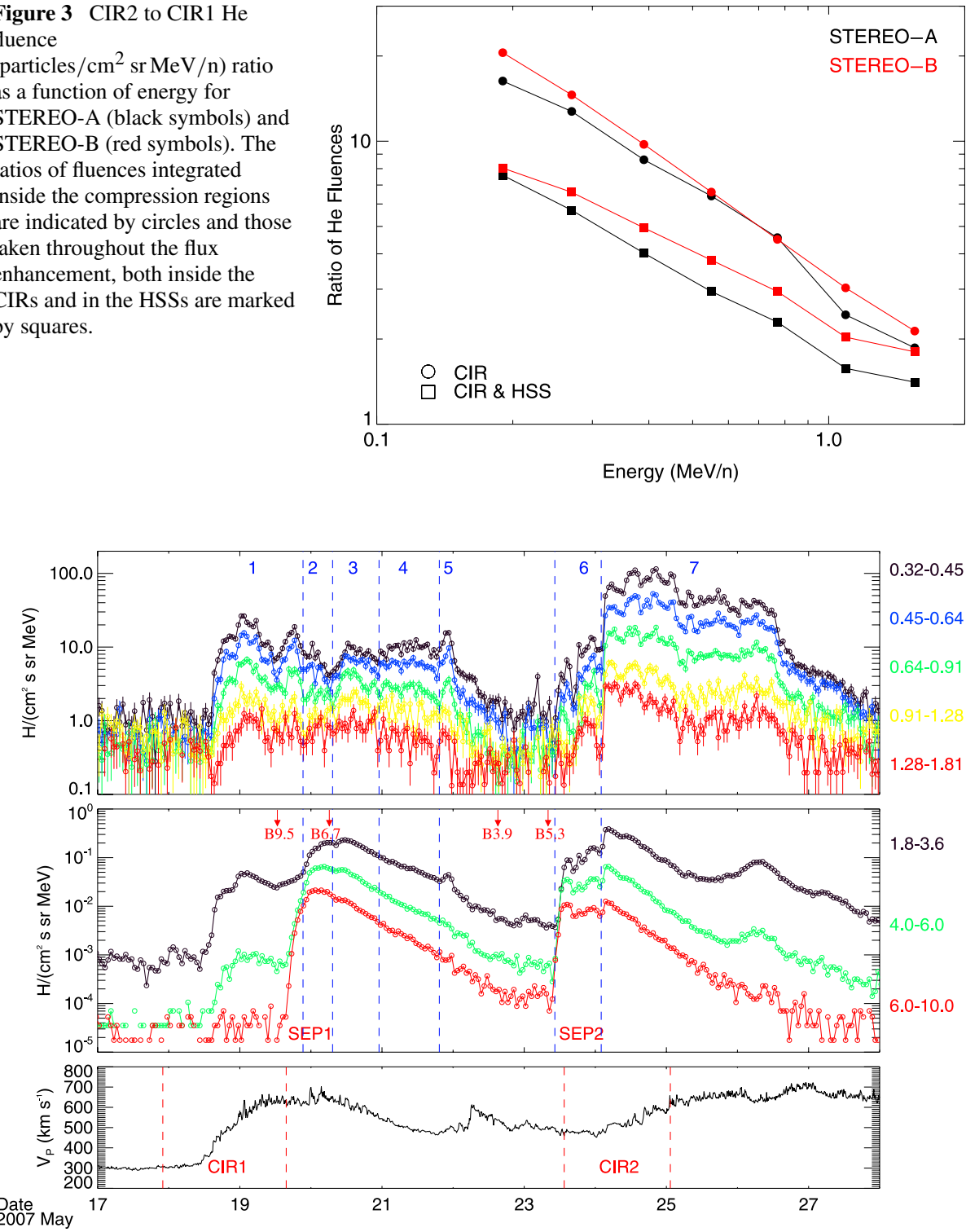

Figure 4 Hourly averaged proton intensities between 0.32 and $10 \mathrm{MeV}$ measured by SIT (upper panel) and LET (middle panel) on STEREO-A. The vertical dashed lines in the proton panels separate time intervals, labeled from 1 to 7 (see text). Downward pointing red vertical arrows in the mid panel indicate solar flares. Bottom panel: The solar wind speed $V_{\mathrm{p}}$.

The dashed vertical lines superimposed on the proton panels mark time intervals (numbered from 1 to 7), where the SIT H intensities are believed to be dominated by contributions from different ion populations. The increases in the time period (1), with peaks inside CIR1 and in the HSS, were observed up to energy of $\sim 6 \mathrm{MeV}$ and are presumably CIR related. The SEP event, identified by the proton intensity onset in the energy range $6-10 \mathrm{MeV}$, at 
16:30 UT on 19 May (hereafter SEP1) was associated with a B9.5 flare at W05, and with a GOES soft X-ray flux maximum at 13:02 UT on 19 May.

We find that the low energy protons measured by SIT show no clear response to the SEP1 event. Around the intensity maximum of $6-10 \mathrm{MeV}$ protons, in time period (2), the proton fluxes in the SIT energy channels continued to decrease or remained flat, indicating that CIR low-energy ions still dominate in the period (2). A later occurring B6.7 flare at 05:56 UT on 20 May originating from the same active region also injected particles into the interplanetary medium (Dröge et al., 2008), and may cause an additional rise of ion fluxes at all energies observed in the period (3). In period (4), the high energy proton fluxes decreased gradually, while at lower energies $(<1.28 \mathrm{MeV})$ they remained essentially constant. We assume that in this interval a connection to the CIR1 reverse shock still exists. At the end of 21 May, marked by (5), the proton intensity below $3.6 \mathrm{MeV}$ showed a minor increase, probably associated with an interplanetary (IP) shock, that passed by the STEREO-A S/C at 01:59 UT on 22 May (listed in Level 3 data). Another small flare (B3.9 class) peaked at 14:47 UT on 22 May and was accompanied with $<100 \mathrm{keV}$ electron event observed by EPAM instrument on ACE satellite. The clear signatures were not observed by SIT or LET instruments since the ion fluxes were very low.

Small ion flux increases in the SIT energy channels within period (6) were related to the next SEP event (hereafter SEP2), with a 6-10 MeV proton intensity onset at 10:30 UT on 23 May, and associated with the B5.3 flare at W51 that peaked at 07:32 UT. The enhancements in period (6) occurred inside the CIR2, near its leading edge. In the period (7) the SEP2 event shows the typical decay phase in the highest measured energy channel. This decay is accompanied by a low energy increase in protons observed with SIT. We claim that this increase is due to the CIR2 event. In the next section abundance measurements will be used to support this claim. The measurements on STEREO-B (not presented) show similar intensity time profiles.

The solar flare identification was obtained from the Solar Geophysical Data. ${ }^{2}$ In association with the flares observed on 19, 22 and 23 May the type II radio emissions were reported, which are the signatures of a shock acceleration process occurring in the gradual events (e.g., Mason, Gloeckler, and Hovestadt, 1984). Throughout the measuring period the STEREO-A (B) S/C were magnetically connected to the solar longitude of $\sim$ W50 (W46).

\subsection{SEP versus CIR Enhancements}

Although the ion enhancements measured by SIT on 24 May appeared late $(\sim 16 \mathrm{hr}$, after onset of the SEP2 event), the question remains whether these enhancements are related to the SEP2 event or not. Reames, Barbier, and Ng (1996) explored SEP events which show sudden increases in ion flux intensity that occur quite late in the events. They suggested that such a behavior can occur either due to an improvement in the magnetic connection between the location of the observer and a stronger acceleration region or the sudden encountering a flux tube filled with particles.

Figure 5 shows STEREO-A and B $\mathrm{H}$ and He ion intensities in the $0.32-0.45 \mathrm{MeV} / \mathrm{n}$ energy channel averaged over $10 \mathrm{~min}$ for a period in 23-26 May 2007. The approximate time delay of $\sim 2 \mathrm{hr}$ between intensity onsets on the A and B satellites on 24 May is marked by two vertical dashed lines. The increase was observed first by STEREO-B and later by STEREO-A. This is consistent with the expected corotation order for an IP magnetic field

\footnotetext{
${ }^{2}$ http://sgd.ngdc.noaa.gov/sgd/jsp/solarindex.jsp.
} 

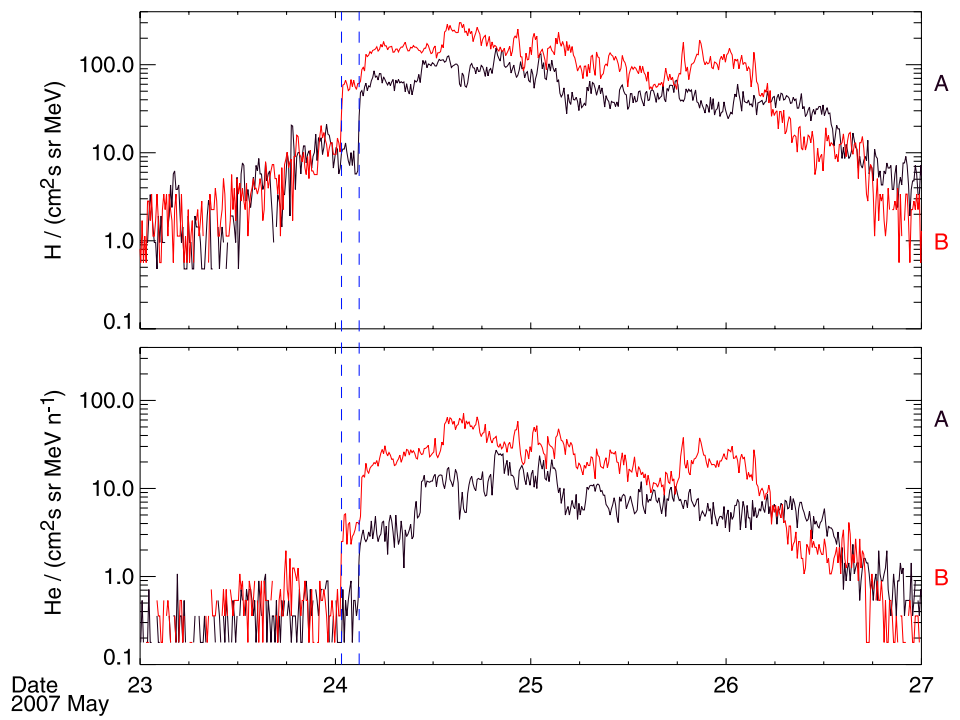

Figure 5 10-min averaged $\mathrm{H}$ (upper panel) and $\mathrm{He}$ (bottom panel) intensities at $0.32-0.45 \mathrm{MeV} / \mathrm{n}$, measured by SIT on STEREO-A (black) and STEREO-B (red) for period 23-26 May 2007. The time delay between the intensity onsets on spacecraft A and B on 24 May is marked by two vertical dashed lines.

line connected to the CIR source region. In addition, the reverse situation was observed for the onset of the $6-10 \mathrm{MeV}$ protons in the SEP2 event. Mewaldt et al. (2008b) reported that for the 23 May 2007 SEP event the protons arrived more than 1 hour later on STEREO-B. Mewaldt et al. (2008b) pointed out that highly variable solar wind structures, provided by the passage of the ICME, organize the SEP data in the 23 May 2007 event. If CIR associated, the particle fluxes would be much more pronounced at larger distances from the Sun due to the positive radial gradients of CIR fluxes reported by Christon and Simpson (1979). Indeed, as shown in Figure 5, the ion fluxes observed by STEREO-B, located on 0.1 AU onward from A, are higher than the fluxes on STEREO-A starting on 24 May. On 26 May, ion fluxes on B dropped below the intensity measured aboard spacecraft A. Note, on 23 May during the SEP2 event the ion fluxes on the A and B satellites were on the average equal.

\subsection{Relative Ion Abundances}

Relative ion abundances can be used to identify the origin of particular ion populations. Mason et al. (1997, 2008a) published $\mathrm{He} / \mathrm{H}, \mathrm{Fe} / \mathrm{O}$ and $\mathrm{He} / \mathrm{O}$ abundance ratios for the CIR events at the energies comparable to the energies in our survey. The $\mathrm{He} / \mathrm{H}, \mathrm{Fe} / \mathrm{O}$ and $\mathrm{He} / \mathrm{O}$ ratios for the gradual SEP events were determined in the study of Mazur et al. (1993) and Desai et al. (2006), respectively. The Fe abundance in the impulsive SEP (ISEP) events were obtained form the survey of Mason et al. (2002).

\subsubsection{He/H Ratio}

In the upper panel of Figure 6 we show hourly proton intensities measured by SIT onboard of STEREO-A in three energy channels $(0.39,0.55,0.77 \mathrm{MeV})$. The lower panels display sixhour $\mathrm{He} / \mathrm{H}$ ratios at the corresponding energies with the error bars indicating the statistical 


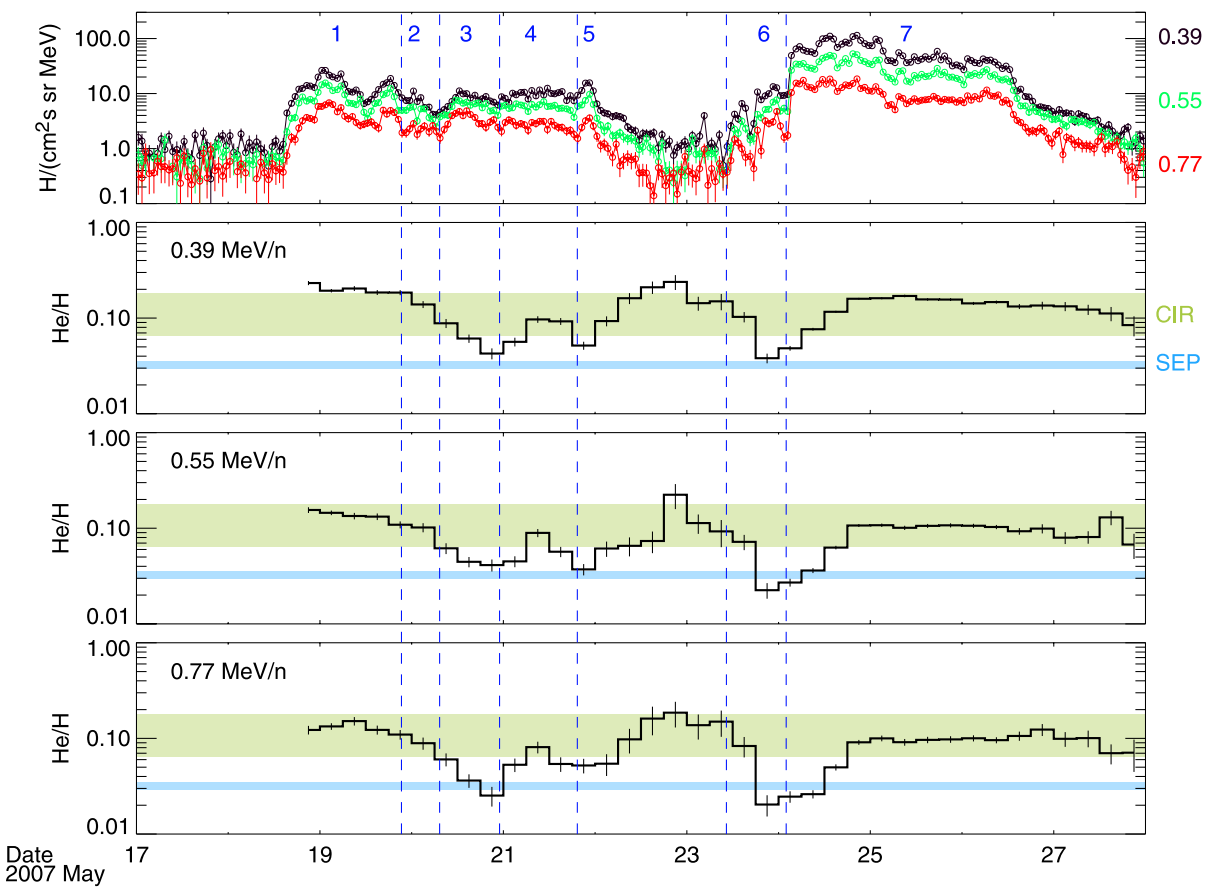

Figure 6 Upper panel: Same as upper panel in Figure 4, but only at energies between 0.32 and $0.91 \mathrm{MeV}$. Panels 2-4: six-hour averaged $\mathrm{He} / \mathrm{H}$ ratio at $0.32-0.45,0.45-0.64$, and $0.64-0.91 \mathrm{MeV} / \mathrm{n}$. Shaded areas show ranges of $\mathrm{He} / \mathrm{H}$ ratios for various particle populations present in the heliosphere: CIR events (green); and gradual SEP events (blue).

uncertainties. The shaded areas present the ranges of $\mathrm{He} / \mathrm{H}$ abundance ratios for the list of CIR events compiled by Mason et al. (1997) and the list of gradual SEPs events compiled by Mazur et al. (1993). The ratio of $\mathrm{He} / \mathrm{H}$ in the investigated period varies from about 0.2 to 0.02 as shown in Figure 6. The highest values were found in the periods (1) and (7), indicating the locations where CIR ions likely dominate. The ratios decreased by a factor of 3 to 5 in the periods (3) and (6), and at the beginning of period (7) where SEP event ions may have contributed. Leske et al. (2008) also reported decreasing $\mathrm{He} / \mathrm{H}$ ratios at $1.8-$ 3.6 MeV/n from measurements by LET instrument on STEREO-A.

Inside the CIR1 region (period 1), the $\mathrm{He} / \mathrm{H}$ ratio remained between 0.1 and 0.2 , close to a CIR event ratio of $0.125 \pm 0.061$ for $0.15 \mathrm{MeV} / \mathrm{n}$ (Mason et al., 1997) or $0.088 \pm 0.011$ for $>2 \mathrm{MeV} / \mathrm{n}$ (Reames, 1995). The ratio was also near a value of $\mathrm{He} / \mathrm{H} \sim 0.15$ measured at solar wind suprathermal energies $(10-35 \mathrm{keV} / \mathrm{n})$ in CIR events by Chotoo et al. (2000). $\mathrm{The} \mathrm{He} / \mathrm{H}$ ratio reached the CIR-like values near the CIR2 trailing edge and remained relatively constant in the fast solar wind. These findings confirm that ion enhancements below $\sim 1 \mathrm{MeV} / \mathrm{n}$ which started on 18 May and 24 May originated from the compression regions CIR1 and CIR2, respectively.

Further we find in this study that during CIR1 and CIR2 events the $\mathrm{He} / \mathrm{H}$ ratio deviates from the range which is given by Mason et al. (1997) for CIR events. The first deviation of the $\mathrm{He} / \mathrm{H}$ ratio occurs at the end of period (3) during the decay phase of the SEP1 event, where the ratio reaches its minimum. The determined ratios are $0.043,0.041$, and 0.025 for energies $0.39,0.55$, and $0.77 \mathrm{MeV} / \mathrm{n}$, respectively. These values are close to the SEP 
event ratio of $0.032 \pm 0.003$ for $>0.3 \mathrm{MeV} / \mathrm{n}$ (Mazur et al., 1993) or $0.036 \pm 0.005$ for $1-4 \mathrm{MeV} / \mathrm{n}$ (Reames, 1995). Later, within period (4), when SEP1 event weakens, the ratio increased to the CIR-like values. It is interesting that around the SEP1 event intensity peak, in the time period (2), the ratio showed only a minor decrease, still remaining near the CIR abundances. The second deviation happens at the beginning of period (7), where the $\mathrm{He} / \mathrm{H}$ ratio remained around the known SEP event ratios. We want to know whether the $\mathrm{He} / \mathrm{H}$ ratios observed in periods (3) and (7) are caused either by direct SEP contamination from the solar events or whether the CIR itself energized the lower energy particle component from the SEP events.

In the middle of the period (6), after the rising phase of the SEP2 event, the ratio dropped for the selected energy intervals to $0.038,0.023$, or 0.021 , close to the SEP event like values. The presence of the IP shock ions, marked by (5), is also accompanied by the decrease of $\mathrm{He} / \mathrm{H}$ ratio towards the SEP event values.

\subsubsection{Fe/O Ratio}

Figure 7 shows six-hour averages of $\mathrm{Fe} / \mathrm{O}$ abundance ratios in three energy channels between 0.06 and $0.16 \mathrm{MeV} / \mathrm{n}$. The red circles indicate ratios with statistical error greater than $35 \%$. Up to period (2) no oxygen nor iron ions were recorded by SIT. Small intensities in those heavy ions are seen at later times. For reference, hourly proton SIT intensities at $0.39 \mathrm{MeV}$ are shown in the upper panel. The shaded areas present the ranges of $\mathrm{Fe} / \mathrm{O}$ ratios for the CIR (Mason et al., 1997), gradual SEP (Desai et al., 2006), and ${ }^{3} \mathrm{He}$-rich or ISEP (Mason et al., 2002) events, respectively. Lower panel shows hourly averaged electron intensities at 38-53 keV measured by EPAM instrument on ACE spacecraft. Note on ACE/EPAM data gap during the period 18-21 May 2007.

Figure 7 shows that the $\mathrm{Fe} / \mathrm{O}$ abundance ratio was around the values found in the literature for ISEP or SEP events on 22-23 May at times of the ICME transit. An obvious ACE/EPAM energetic electron event on 22 May 2007 points to the SEP injections as responsible for the Fe ions we see. Note the most prominent feature in the electron panel is the increase on 23 May 2007 associated with the SEP2 event. A possible explanation for the Fe enhancement during the ICME (period 5) could be the presence of impulsive flare particles inside the ICME (see Mazur et al., 1998). The ICME magnetic field connects to the active region at the Sun, which continues in production of the flare on 22 May 2007. This could also be the explanation for the enhanced He abundance during the ICME passage as shown in Figure 6. The impulsive events are known to have a high $\mathrm{He} / \mathrm{H}$ ratio when compared with corresponding values in gradual events (Reames, 1990). The Fe/O ratio close to the ISEP/SEP value in the period (6) was associated with the SEP2 event. Similarly to the $\mathrm{He} / \mathrm{H}$ ratio the Fe/O was still near the SEP-like ratios at beginning of period (7). Near noon of 24 May (still inside CIR2), when the SEP2 event continued to decline, the Fe/O approached the CIR-like ratio and remained around this value outside the compression region.

Figure 8 shows STEREO-A SIT mass histograms with counts accumulated during $24 \mathrm{hr}$ on 24 May 2007 in the energy bands $0.45-0.64 \mathrm{MeV} / \mathrm{n}$ for $\mathrm{H}-\mathrm{He}$ (left panel) and 0.08 $0.11 \mathrm{MeV} / \mathrm{n}$ for $\mathrm{He}-\mathrm{Fe}$ (right panel). The blue (red) color marks mass ranges defined for $\mathrm{H}\left({ }^{4} \mathrm{He}\right)$ and $\mathrm{O}(\mathrm{Fe})$ ions. All ion peaks used in the determination of the abundances are clearly resolved. Note that in the $0.08-0.11 \mathrm{MeV} / \mathrm{n}$ energy range the He ions may still have energies below the instrument threshold energy. Therefore the He peak in the right panel is smaller than the O peak. 

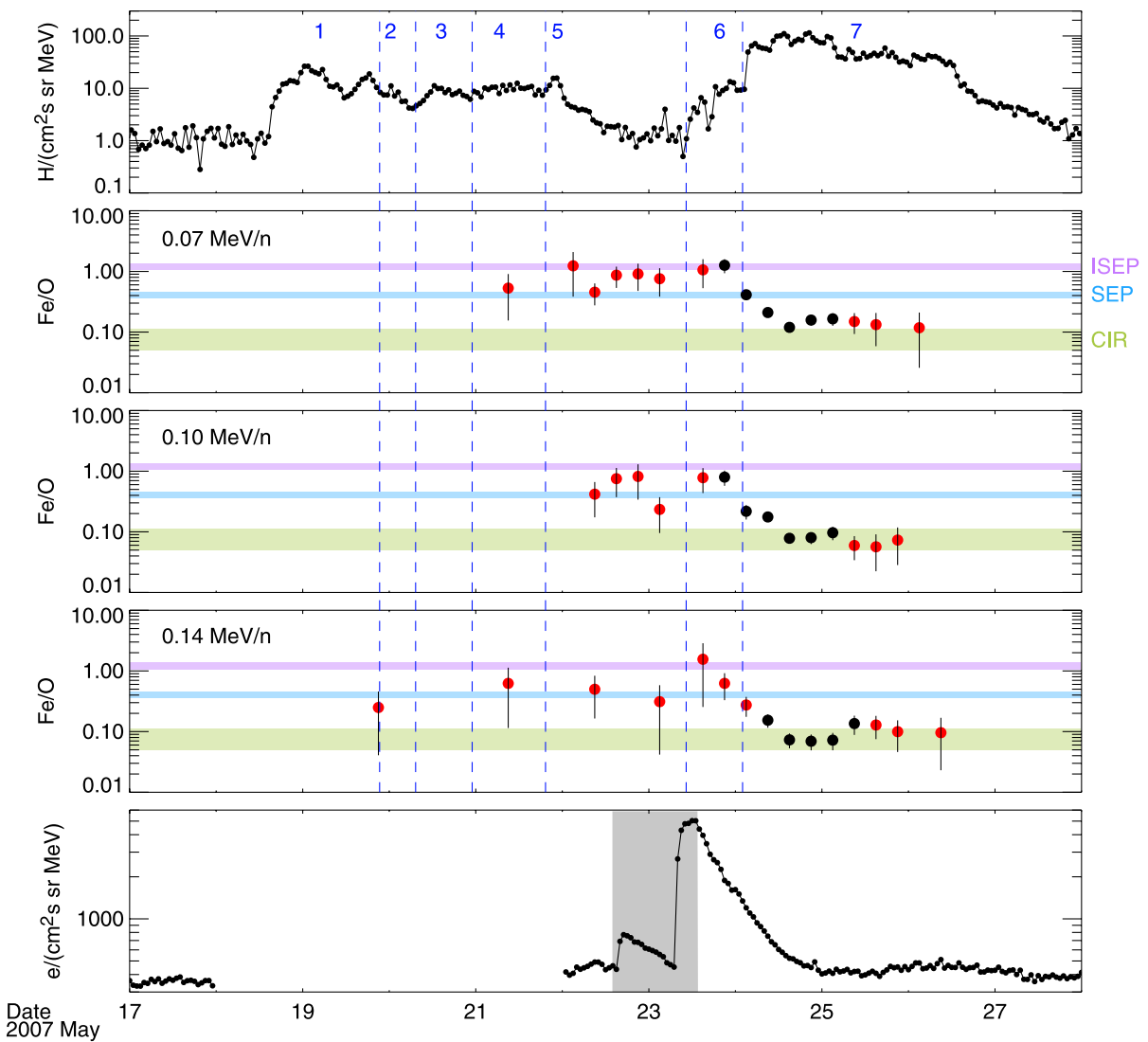

Figure 7 Upper panel: Same as upper panel in Figure 4, but only at 0.32-0.45 MeV. Panels 2-4: six-hour averaged $\mathrm{Fe} / \mathrm{O}$ ratio at $0.06-0.08,0.08-0.11$, and $0.11-0.16 \mathrm{MeV} / \mathrm{n}$. The red circles indicate ratios with statistical uncertainty greater than $35 \%$. Shaded areas show ranges of $\mathrm{Fe} / \mathrm{O}$ ratios for various particle populations present in the heliosphere: CIR events (green); Gradual SEP events (blue); and ${ }^{3} \mathrm{He}$-rich or ISEP events (violet). Lower panel: ACE/EPAM hourly electron intensities at $38-53 \mathrm{keV}$. The gray shaded region marks the time interval of the ICME.

\subsection{3. $\mathrm{He} / \mathrm{O}$ Ratio}

Figure 9 shows six hours of $\mathrm{He} / \mathrm{O}$ abundance ratios in three energy channels between 0.11 and $0.32 \mathrm{MeV} / \mathrm{n}$. The figure is in the same format as Figure 7 for $\mathrm{Fe} / \mathrm{O}$ ratio. The shaded areas present the ranges of $\mathrm{He} / \mathrm{O}$ ratios for CIR (Mason et al., 2008a) and gradual SEP events (Desai et al., 2006).

Similarly to $\mathrm{He} / \mathrm{H}$ the $\mathrm{He} / \mathrm{O}$ ratio was around the CIR-like values inside the period (1) and in the fast solar wind in period (7). The He/O also decreased to the SEP event-like values in the periods $(2-3)$ but due to higher statistical errors the declining trend with the ratio minimum in the end of the period (3) was not seen here. Figure 9 shows that during the $\mathrm{SEP} 2$ event onset (period 6) the $\mathrm{He} / \mathrm{O}$ ratio at two energies, 0.19 and $0.27 \mathrm{MeV} / \mathrm{n}$, reached the SEP event ratio. At lower energy $(0.14 \mathrm{MeV} / \mathrm{n})$ the ratio was close to the CIR like values and did not reach the SEP He/O reference values shown for the energies of $0.38 \mathrm{MeV} / \mathrm{n}$. While there were observed SEP-like $\mathrm{Fe} / \mathrm{O}$ and $\mathrm{He} / \mathrm{H}$ abundances at the beginning of the $\mathrm{CIR} 2$ event, the $\mathrm{He} / \mathrm{O}$ ratio showed the typical corotating values. 

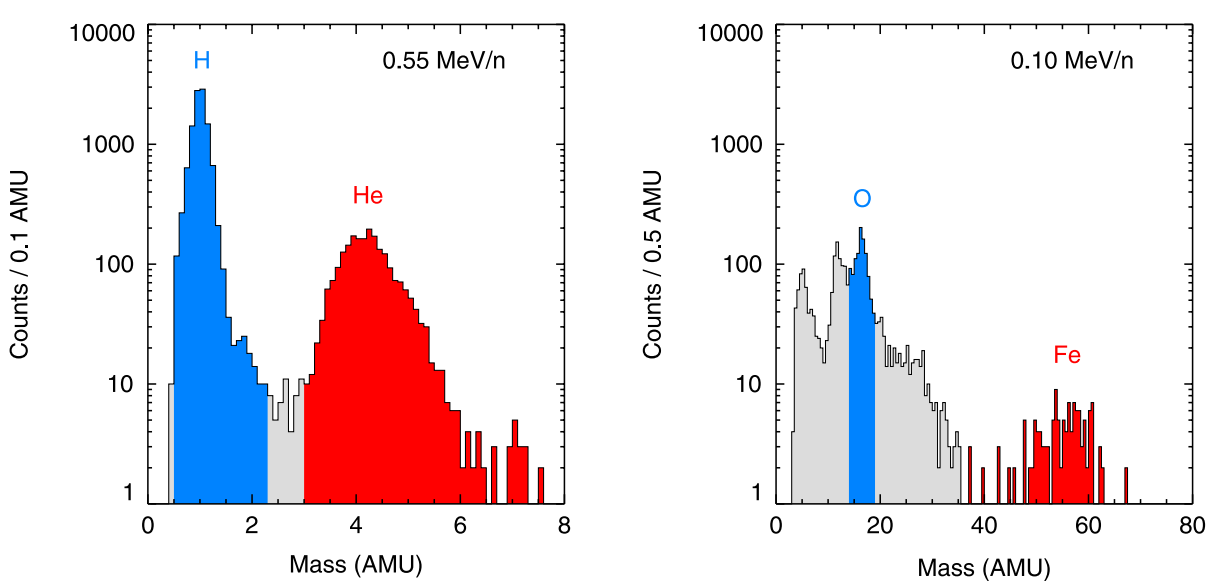

Figure 8 STEREO-A SIT mass histograms on 24 May 2007 for $0.45-0.64 \mathrm{MeV} / \mathrm{n}$ (left) and $0.08-0.11 \mathrm{MeV} / \mathrm{n}$ (right).

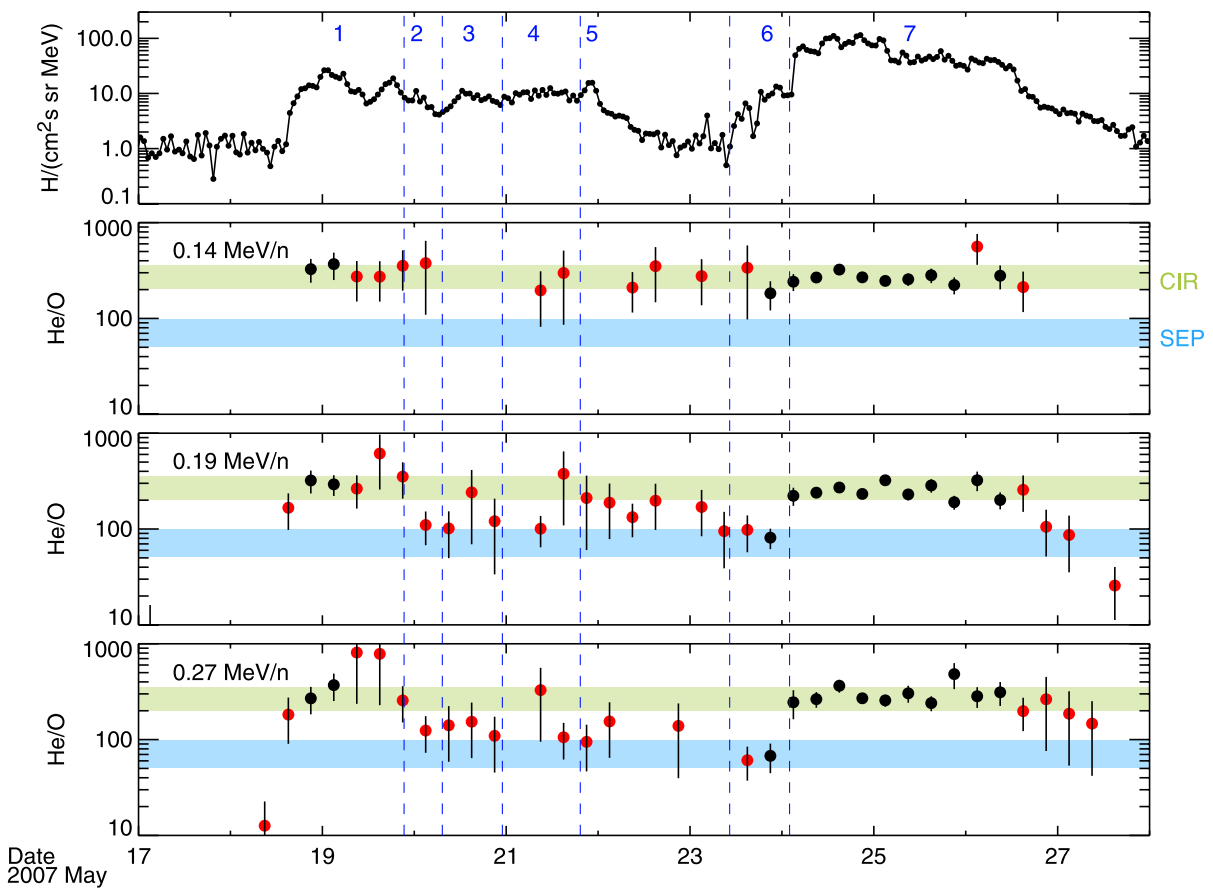

Figure 9 Upper panel: Same as upper panel in Figure 4. Panels 2-4: six-hour averaged He/O ratio at $0.11-0.16,0.16-0.23$, and $0.23-0.32 \mathrm{MeV} / \mathrm{n}$. The red circles indicate ratios with statistical uncertainty greater than $35 \%$. Shaded areas show ranges of $\mathrm{He} / \mathrm{O}$ ratios for various particle populations present in the heliosphere: CIR events (green); and gradual SEP events (blue).

It is noted that while $\mathrm{Fe} / \mathrm{O}$ is enhanced by a factor of $\sim 5$ in the SEP events compared to the CIR events the $\mathrm{He} / \mathrm{O}$ is decreased by a factor of $\sim 3$. This may explain why there was no corresponding decrease observed in the $\mathrm{He} / \mathrm{O}$ ratio to the SEP-like values as it 

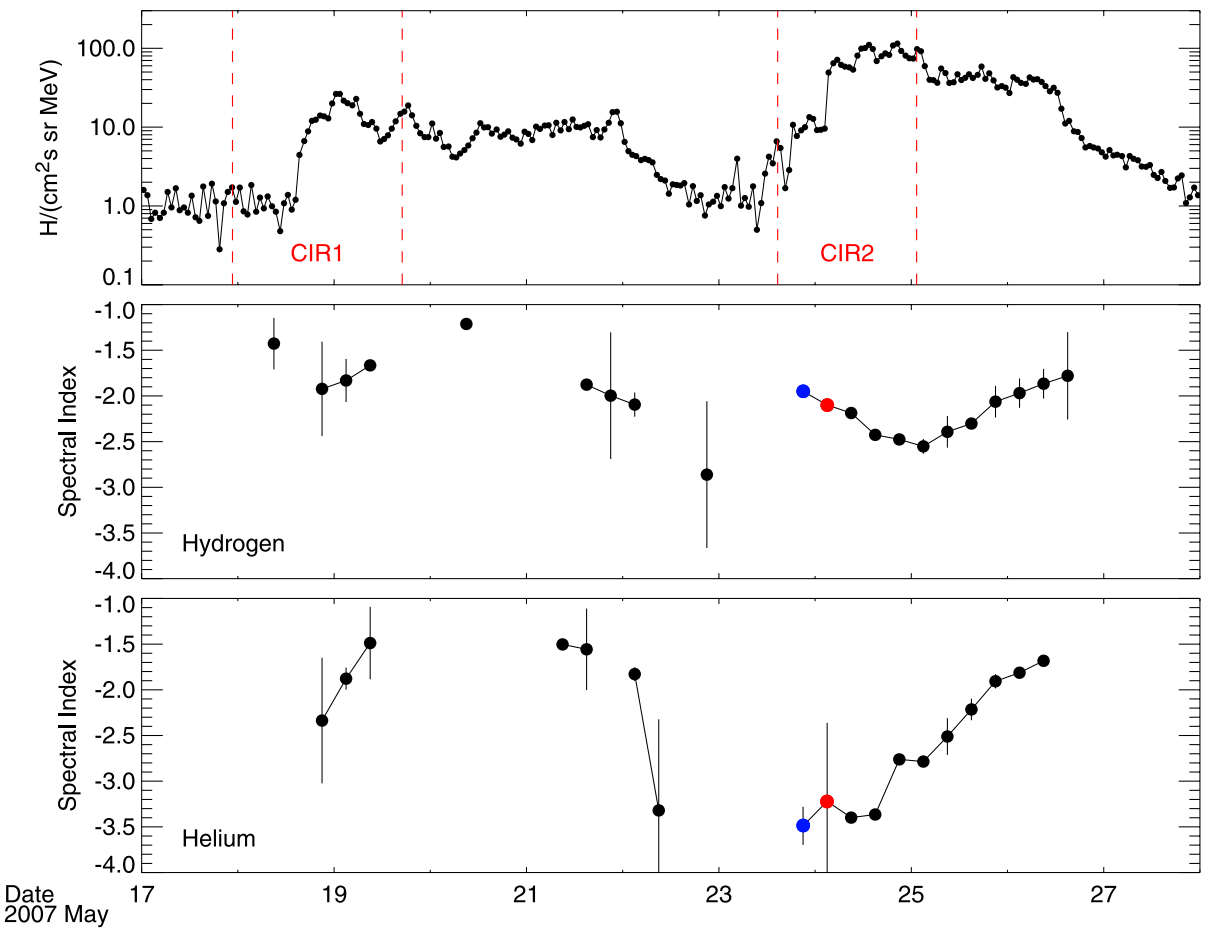

Figure 10 Upper panel: Same as upper panel in Figure 7. Panels 2 and 3: six-hour $\mathrm{H}$ and He spectral indices at $0.32-1.81 \mathrm{MeV}$ and $0.16-0.91 \mathrm{MeV} / \mathrm{n}$, respectively. Only indices with statistical error less than $35 \%$ are plotted. The spectra at the end of period (6) and at the beginning of period (7) are marked by blue and red, respectively.

was for Fe/O during the CIR2 event. The observations by Malandraki et al. (2007, 2008) during the periods of CIR/SEP combinations similarly show CIR abundance of the He/O ratio whereas $\mathrm{C} / \mathrm{O}$ was consistent with SEP values. They suggested that since there is not much $\mathrm{C}$ production beyond $3 \mathrm{AU}$ the CIR might have re-accelerated $\mathrm{C}$ ions from the SEP population.

\subsection{Energy Spectra}

Figure 10 shows spectral indices obtained from fitting power laws in energy to $\mathrm{H}$, and $\mathrm{He}$ intensities over the range $0.32-1.81 \mathrm{MeV} / \mathrm{n}$ and $0.16-0.91 \mathrm{MeV} / \mathrm{n}$ for each 6 -hr time interval between 17 May and 27 May 2007. Only spectral indices with statistical errors less than $35 \%$ are included in the figure. One-hour $\mathrm{H}$ intensities at $0.32-0.45 \mathrm{MeV}$ are shown for reference in the upper panel. The SEP2 event spectral index at 18-24 UT on 23 May, and CIR2 event spectral index at 00-06 UT on 24 May are marked by blue and red circles, respectively.

The $\mathrm{H}(\mathrm{He})$ spectral index associated with the SEP2 event, $-1.95 \pm 0.05(-3.49 \pm$ $0.21)$, showed only a little change, by $\leq 0.27$ units, to $-2.10 \pm 0.01(-3.22 \pm 0.86)$ over the next six hours, even though related fluxes suddenly rose in all energy channels (see Figure 4, upper panel). Figure 10 shows clearly that the proton spectrum inside CIR2 softens moderately while the He spectrum does not change much. An exception occurs when the 


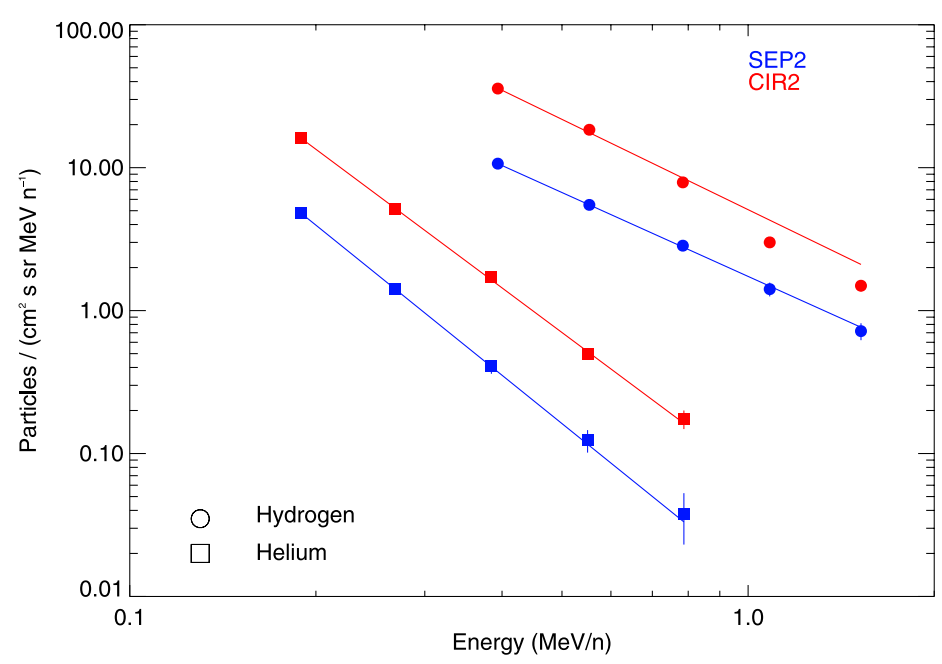

Figure 11 Energy spectra of $\mathrm{H}$ (circles), and He (squares) during the six-hour intervals at the end of period (6), marked by SEP2, and at the beginning of period (7), marked by CIR2. The lines are power-law fits.

spectrum hardens near the trailing edge. In the fast solar wind both spectra gradually harden. The hardening of the corotating spectra with time has been noted in previous surveys and could either be a propagation effect or a change in the source spectra (see Mason et al., 1999).

Figure 11 shows differential energy spectra related to the SEP2 and CIR2 events, discussed in the previous paragraph. The circles correspond to $\mathrm{H}$ and squares correspond to $\mathrm{He}$ intensities. The lines in the figure denote least-squares fits to the observations. The $\mathrm{He}$ spectra show a clear power-law shape over the narrow energy range in our observations. While the SEP2 event $\mathrm{H}$ spectrum shows a power-law shape, the CIR2 event $\mathrm{H}$ spectrum changes around $1 \mathrm{MeV} / \mathrm{n}$. The power law spectral shape below $1 \mathrm{MeV} / \mathrm{n}$ and the steepening of the spectrum above $1 \mathrm{MeV} / \mathrm{n}$ have been reported for $\mathrm{H}$ and He CIR ions (Mason et al., 1999 and references therein).

The average energy spectral slope inside the CIR2 compression region at 00-24 UT 24 May is $-2.30 \pm 0.01$ for $\mathrm{H}$ and $-3.19 \pm 0.22$ for He. The corresponding values derived inside CIR1 between 18:00 UT on 18 May and 12:00 UT on 19 May are much harder, $-1.81 \pm 0.19$ for $\mathrm{H}$ and $-1.90 \pm 0.27$ for He. Similar spectra with a power-law index $\sim-2$ are also found for $0.54-1.85 \mathrm{MeV}$ protons at corotating reverse shocks in the outer heliosphere (Barnes and Simpson, 1976). Below $\sim 1 \mathrm{MeV} / \mathrm{n}$ the spectral forms of CIR heavy ions are fitted with a power-law index of $-2.51 \pm 0.10$ (Mason et al., 2008a). The energy spectra of heavy ions from SEP events are typically harder with slopes ranging from -1.0 to - 1.5 (Mason et al., 2002; Mewaldt et al., 2005).

\section{Discussion}

\subsection{CIR2 Event}

As pointed out by Scholer, Morfill, and van Hollebeke (1980) and Desai et al. (1998) the intensity of particles accelerated at the corotating shocks is determined only by the intensity 
of the seed particles and the acceleration strength of the shock. In Section 2.1 we have shown that the speed difference between high and slow solar wind, probably related to the shock strength (Kobayashi et al., 2000), was less enhanced in the CIR2 region. A similar feature is seen in the total pressure and the magnetic field magnitude. From these observations we assume that the acceleration strength of the CIR2 region beyond 1 AU may not be higher than that of the CIR1 compression region. More detailed analysis of the IP magnetic field and plasma parameters is clearly needed to provide further support for such interpretation. However, a harder $\mathrm{H}$ and $\mathrm{He}$ spectrum found in the CIR1 compared to the spectra in the CIR2 may suggest a higher acceleration strength for the CIR1 compression region, favoring the above assumption. One would expect that if the shock strengths of the CIR1 and CIR2 regions are even similar and the higher intensities are observed in association with the CIR2, then the CIR2 compression region accelerate particles out of seed populations with higher intensities.

Based on the above arguments, we consider the following scenario that may account for the observations shown in Figure 3. The overabundance of the He fluences associated with CIR2 and its power-law decrease below $\sim 1 \mathrm{MeV} / \mathrm{n}$ probably reflects an additional source particle population which does not contribute to the CIR1 acceleration. We note that the time interval between the arrival of the trailing edges of the adjacent compression regions of CIR1 and CIR2 was $~ 5.5$ days. Desai et al. (1998) assumed that two adjacent compression regions observed during a single $\sim 26$-day period accelerate the same seed population provided that there are no solar transients. Small overabundance of the He fluence at higher energies, which is not dependent on energy in a simple way, is likely due to contamination with the SEP2 event ions.

As pointed out earlier, the shock strength alone may not play a dominant role in determining the intensity. Other effects may be important including the merging of CIRs in the outer heliosphere, the latitudinal extent of the CIR shocks (Richardson et al., 1993), and the connection to the CIR (Mason et al., 2009). The tendency for the higher peak intensities associated with the recurrent CIRs was seen in a survey by Mason et al. (2008a) and Richardson et al. (1993). Mason et al. (2008a) also noted that larger coronal holes might produce parent shock structures of larger size which are responsible for the higher intensities. An examination of the SIT PHA data in the previous CRs reveals that the CIR2 event had not been present for a long time. This event appears as a very faint increase only in one solar rotation preceding the CR 2056 (see Figure 1). Furthermore, no big difference in size of parent coronal holes responsible for the CIR1 and CIR2 events has been found, as inferred from the regions of low emission in the STEREO-A SECCHI solar images at EUV $171 \AA$ (Li et al., 2008; Figure 1).

Since the SEP2 event is a western hemisphere event, the typical shape of the intensity time profile should include the rapid rise and decline (Reames, Barbier, and $\mathrm{Ng}, 1996$ ). A rather complex intensity - time profile has been observed at the highest energies with a rapid rise and a plateau in the period (6), and a decline in the period (7) (see Figure 4, middle panel). Despite this it is likely that the SEP2 ion fluxes, as measured by SIT, attained peak values within period (6) and continued to decay in period (7). Below $\sim 1 \mathrm{MeV} / \mathrm{n}$ the $\mathrm{H}$ and He intensities in the period (6) are about one order of magnitude lower than the intensities at the beginning of period (7). Therefore, it would be difficult for SEP event ions with the intensities observed in (6) to substantially affect the CIR elemental composition in period (7). Rather, an observed SEP-like composition of $\mathrm{H}, \mathrm{He}, \mathrm{O}$, and Fe ions inside CIR2 may result from energizing low-energy, i.e., more abundant solar flare ions by the compression region. Late in the SEP2 event, such particles are already filling the region beyond the observation point and may therefore be available for an acceleration by the CIR-shocks. Such a conclusion is further supported by the similarity of the corotating and the preceding solar event 
spectra. The ratio of the CIR to SEP spectral index is 1.08 for hydrogen and 0.92 for helium, close to unity, as would be predicted by Axford (1981), if CIR-shock accelerated particles were energized out of an SEP population with a flatter spectrum than the CIR shock would produce. An assumption of flatter spectrum in the SEP events is consistent with the observations. Kocharov et al. (2003) showed that compression regions associated with CIRs near 1 AU could re-accelerate solar energetic particles.

The differential energy spectra shown in Figure 11 allow a rough estimate of the energy gain of compression region accelerated solar particles. For example, the intensity of solar event protons at $0.4 \mathrm{MeV}$ is equal to the intensity of the CIR associated protons at $0.7 \mathrm{MeV}$ as found by the fitted energy spectra. On average, the $0.4-0.9 \mathrm{MeV}$ solar event protons gain a factor of $\sim 1.7$ in energy and the $0.2-0.5 \mathrm{MeV} / \mathrm{n}$ solar helium ions gain a factor of $\sim 1.5$. These values are somewhat lower than a factor of two for IP shock accelerated solar flare particles reported in a survey of Tan et al. (1989). In our study, the assumed seed and energized particles are, however, measured locally at 1 AU while the CIR acceleration should take place beyond $2 \mathrm{AU}$. It is known that flare fluxes decrease roughly as $\sim r^{-3}$ (Parker, 1963, p. 211), which decreases the shock seed population at the acceleration site at larger radial distances. At the same time, accelerated particles, while streaming back to $1 \mathrm{AU}$, should experience an adiabatic cooling in the expanding solar wind (Fisk and Lee, 1980), which decreases the flux measured in a given energy window at 1 AU. These two effects may result in an underestimation of our inferred energy gain, due to the fact that we have used a stronger seed population along with the weaker accelerated population.

Our observations made on both STEREO spacecraft reveal that He ion fluxes at lower energies peak within the CIR, while at higher energies the ion fluxes in the HSS become dominant. The presence of the ion intensity peaks inside the compression regions brings up the question on their origin, since these fluxes are not supposed to be connected with the distant shocks in the outer heliosphere. A non-field-aligned transport is required to populate field lines inside a CIR and/or other non-shock processes within a CIR may contribute (Richardson, 2004 and references therein).

\subsection{CIR1 Event}

At the time of maximum intensity of 6-10 MeV protons (20 May, 01:00 UT) the solar wind speed of $\sim 650 \mathrm{~km} \mathrm{~s}^{-1}$ translates into 1.01 AU Archimedean spiral path length for the STEREO-A S/C located at $0.96 \mathrm{AU}$. Assuming that the particles would propagate scatter free along an interplanetary magnetic field spiral with zero pitch angle, the expected intensity maximum of the $0.6 \mathrm{MeV} / \mathrm{n}$ solar ions, would be delayed by $3 \mathrm{hr}$ with respect to the $6-10 \mathrm{MeV}$ intensity peak. However, the $\mathrm{He} / \mathrm{H}$ ratio has reached the nearest value close to the SEP-like values at $\sim 21: 00$ UT on 20 May (see Figure 6 ), $\sim 17 \mathrm{hr}$ after the expected flux maximum.

A possible explanation for our observations could be that the re-acceleration of the low energy solar particles from the SEP1 event by the corotating shock in the outer heliosphere led to the SEP-like abundance observed at decaying HSS at the end of 20 May. The contamination of the CIR1 by SEPs would offer a simpler explanation. The SEP1 event is about factor of two more intense than the SEP2 event when we compare the $6-10 \mathrm{MeV}$ proton intensities. Also the $0.39 \mathrm{MeV} / \mathrm{n}$ He peak intensities in the CIR1 event are only $\sim 1 / 4$ of the peak intensities in the CIR2 event. The $\mathrm{He} / \mathrm{H}$ ratio approached SEP-like values in the late phase of the CIR1 event (see Figure 6) when the intensities are much smaller than the peak intensities. Thus, during that phase of the CIR1 event the SEP1 intensities at lower energies might have been comparable or higher than the CIR1 intensities and the contamination may be important. Since there was no clear anisotropy observed in the rising phase 
of the $\sim 100 \mathrm{keV}$ electrons in the SEP1 event (Dröge et al., 2008), the assumption of scatter free propagation may not be valid anymore. The SEP-like low energy $\mathrm{He} / \mathrm{H}$ ratios which appear delayed with respect to the SEP1 event intensity peak may be a consequence of a small scattering mean free path between the Sun and 1 AU. Alternatively, there may be a contamination from solar ions associated with the flare on 20 May, which occurred $\sim 17 \mathrm{hr}$ after the previous solar flare, and which may lead to the $\mathrm{He} / \mathrm{H}$ minimum at the end of period (3). However, particle signatures at the highest measured energies are very weak and are difficult to associate unambiguously to the 20 May flare.

\section{Summary}

We have investigated particle events at energies from $\sim 0.1$ to $\sim 1.0 \mathrm{MeV} / \mathrm{n}$, associated with two compression regions, CIR1 and CIR2, with corotating streams closely located in heliolongitude, which immediately precede and follow two weak SEP event onsets. We suggest that an overabundance of ion fluxes in association of the CIR2 could be due to additional source population. The relative abundances of $\mathrm{H}, \mathrm{He}, \mathrm{O}$, and $\mathrm{Fe}$ inside the CIR2 compression region and $\mathrm{He} / \mathrm{H}$ ratio in the $\mathrm{HSS}$ of $\mathrm{CIR} 1$ region are very close to the solar energetic particle relative abundances found in the flare event in this survey and in previously published solar flare surveys. The similarity in elemental abundances and in spectral slopes suggests that the CIR 2 contains a seed population ions from the preceding SEP event. The elemental abundances late in the CIR1 event can be explained by an SEP contamination.

Acknowledgements We thank B. Heber, B. Inhester, N. Srivastava, M. Fränz, and B. Klecker for helpful discussions. We acknowledge STEREO PLASTIC under NASA Contract NAS5-00132 for the use of solar wind plasma data. STEREO LET data were obtained from http://www.srl.caltech.edu. ACE EPAM data were obtained from the ACE Science Center (http://www.srl.caltech.edu/ACE/ASC/). This work was supported by the Max-Planck-Gesellschaft zur Förderung der Wissenschaften and the Bundesministerium für Bildung und Forschung (BMBF) under grant 50 OC 0501. The work at the Johns Hopkins University/Applied Physics Laboratory was supported by NASA under contract SA4889-26309 from the University of California Berkeley.

Open Access This article is distributed under the terms of the Creative Commons Attribution Noncommercial License which permits any noncommercial use, distribution, and reproduction in any medium, provided the original author(s) and source are credited.

\section{References}

Acuña, M.H., Curtis, D., Scheifele, J.L., Russell, C.T., Schroeder, P., Szabo, A., Luhmann, J.G.: 2008, The STEREO/IMPACT magnetic field experiment. Space Sci. Rev. 136, 203-226. doi:10.1007/s11214-007-9259-2.

Axford, I.W.: 1981, Acceleration of cosmic rays by shock waves. In: 17th International Cosmic Ray Conference 12, $155-203$.

Barnes, C.W., Simpson, J.A.: 1976, Evidence for interplanetary acceleration of nucleons in corotating interaction regions. Astrophys. J. 210, $91-96$.

Belcher, J.W., Davis, L. Jr.: 1971, Large-amplitude Alfvén waves in the interplanetary medium, 2. J. Geophys. Res. 76, 3534-3563. doi:10.1029/JA076i016p03534.

Burlaga, L.F.: 1995, Interplanetary Magnetohydrodynamics. Oxford University Press, New York.

Chotoo, K., Schwadron, N.A., Mason, G.M., Zurbuchen, T.H., Gloeckler, G., Posner, A., Fisk, L.A., Galvin, A.B., Hamilton, D.C., Collier, M.R.: 2000, The suprathermal seed population for corotating interaction region ions at $1 \mathrm{AU}$ deduced from composition and spectra of $\mathrm{H}^{+}, \mathrm{He}^{++}$, and $\mathrm{He}^{+} \mathrm{ob}-$ served on Wind. J. Geophys. Res. 105, 23107-23122. doi:10.1029/1998JA000015.

Christon, S.P., Simpson, J.A.: 1979, Separation of corotating nucleon fluxes from solar flare fluxes by radial gradients and nuclear composition. Astrophys. J. 227, 49-53. doi:10.1086/182865. 
Desai, M.I., Marsden, R.G., Sanderson, T.R., Balogh, A., Forsyth, R.J., Gosling, J.T.: 1998, Particle acceleration at corotating interaction regions in the three-dimensional heliosphere. J. Geophys. Res. 103, $2003-2014$.

Desai, M.I., Mason, G.M., Gold, R.E., Krimigis, S.M., Cohen, C.M.S., Mewaldt, R.A., Mazur, J.E., Dwyer, J.R.: 2006, Heavy-ion elemental abundances in large solar energetic particle events and their implications for the seed population. Astrophys. J. 649, 470-489. doi:10.1086/505649.

Dröge, W., Gómez-Herrero, R., Müller-Mellin, R., Klassen, A., Kartavykh, J., Heber, B., Haggerty, D., Klecker, B.: 2008, Simultaneous ACE/STEREO observations of solar electron events in May 2007. In: 37th COSPAR Scientific Assembly 37, 749.

Fisk, L.A., Lee, M.A.: 1980, Shock acceleration of energetic particles in corotating interaction regions in the solar wind. Astrophys. J. 237, 620-626. doi:10.1086/157907.

Galvin, A.B., Kistler, L.M., Popecki, M.A., Farrugia, C.J., Simunac, K.D.C., Ellis, L., Möbius, E., Lee, M.A., Boehm, M., Carroll, J., Crawshaw, A., Conti, M., Demaine, P., Ellis, S., Gaidos, J.A., Googins, J., Granoff, M., Gustafson, A., Heirtzler, D., King, B., Knauss, U., Levasseur, J., Longworth, S., Singer, K., Turco, S., Vachon, P., Vosbury, M., Widholm, M., Blush, L.M., Karrer, R., Bochsler, P., Daoudi, H., Etter, A., Fischer, J., Jost, J., Opitz, A., Sigrist, M., Wurz, P., Klecker, B., Ertl, M., Seidenschwang, E., Wimmer-Schweingruber, R.F., Koeten, M., Thompson, B., Steinfeld, D.: 2008, The plasma and suprathermal ion composition (PLASTIC) investigation on the STEREO observatories. Space Sci. Rev. 136, 437 - 486. doi:10.1007/s11214-007-9296-x.

Hundhausen, A.J., Gosling, J.T.: 1976, Solar wind structure at large heliocentric distances - an interpretation of Pioneer 10 observations. J. Geophys. Res. 81, 1436-1440.

Kilpua, E.K.J., Liewer, P.C., Farrugia, C., Luhmann, J.G., Möstl, C., Li, Y., Liu, Y., Lynch, B.J., Russell, C.T., Vourlidas, A., Acuna, M.H., Galvin, A.B., Larson, D., Sauvaud, J.A.: 2009, Multispacecraft observations of magnetic clouds and their solar origins between 19 and 23 May 2007. Solar Phys. 254, 325 344. doi:10.1007/s11207-008-9300-y.

Kobayashi, M.N., Doke, T., Kikuchi, J., Hayashi, T., Itsumi, K., Takashima, T., Takehana, N., Shirai, H., Yashiro, J., Hasebe, N., Kondoh, K., Yanagimachi, T., Nagatani, M., Harada, A., Wilken, B.: 2000, The correlation between CIR ion intensity and solar wind speed at 1 AU. Adv. Space Res. 26, $861-864$.

Kocharov, L., Kovaltsov, G.A., Torsti, J., Anttila, A., Sahla, T.: 2003, Modeling the propagation of solar energetic particles in corotating compression regions of solar wind. J. Geophys. Res. 108, 1404. doi:10.1029/2003JA009928.

Leske, R.A., Mewaldt, R.A., Mason, G.M., Cohen, C.M.S., Cummings, A.C., Davis, A.J., Labrador, A.W., Miyasaka, H., Stone, E.C., Wiedenbeck, M.E., von Rosenvinge, T.T.: 2008, STEREO and ACE observations of CIR particles. In: Li, G., Hu, Q., Verkhoglyadova, O., Zank, G.P., Lin, R.P., Luhmann, J. (eds.) Particle Acceleration and Transport in the Heliosphere and Beyond, American Institute of Physics Conference Series 1039, 131 - 136. doi:10.1063/1.2982435.

Li, Y., Lynch, B.J., Stenborg, G., Luhmann, J.G., Huttunen, K.E.J., Welsch, B.T., Liewer, P.C., Vourlidas, A.: 2008, The solar magnetic field and coronal dynamics of the eruption on 2007 May 19. Astrophys. J. 681, 37 - 40. doi:10.1086/590340.

Liu, Y., Luhmann, J.G., Huttunen, K.E.J., Lin, R.P., Bale, S.D., Russell, C.T., Galvin, A.B.: 2008, Reconstruction of the 2007 May 22 magnetic cloud: How much can we trust the flux-rope geometry of CMEs? Astrophys. J. 677, 133 - 136. doi:10.1086/587839.

Malandraki, O.E., Marsden, R.G., Tranquille, C., Forsyth, R.J., Elliott, H.A., Lanzerotti, L.J., Geranios, A.: 2007, Energetic particle observations by Ulysses during the declining phase of solar cycle 23. J. Geophys. Res. 112, 6111. doi:10.1029/2006JA011876.

Malandraki, O.E., Marsden, R.G., Tranquille, C., Forsyth, R.J., Elliott, H.A., Geranios, A.: 2008, Energetic particle measurements from the Ulysses/COSPIN/LET instrument obtained during the August/September 2005 events. Ann. Geophys. 26, 1029-1037.

Mason, G.M., Gloeckler, G., Hovestadt, D.: 1984, Temporal variations of nucleonic abundances in solar flare energetic particle events. II - Evidence for large-scale shock acceleration. Astrophys. J. 280, 902 - 916. doi:10.1086/162066.

Mason, G.M., Mazur, J.E., Dwyer, J.R., Reames, D.V., von Rosenvinge, T.T.: 1997, New spectral and abundance features of interplanetary heavy ions in corotating interaction regions. Astrophys. J. 486, $149-$ 152. doi: $10.1086 / 310845$.

Mason, G.M., von Steiger, R., Decker, R.B., Desai, M.I., Dwyer, J.R., Fisk, L.A., Gloeckler, G., Gosling, J.T., Hilchenbach, M., Kallenbach, R., Keppler, E., Klecker, B., Kunow, H., Mann, G., Richardson, I.G., Sanderson, T.R., Simnett, G.M., Wang, Y.M., Wimmer-Schweingruber, R.F., Fränz, M., Mazur, J.E.: 1999, Origin, injection, and acceleration of CIR particles: Observations report of Working group 6. Space Sci. Rev. 89, 327-367. doi:10.1023/A:1005278214143.

Mason, G.M., Wiedenbeck, M.E., Miller, J.A., Mazur, J.E., Christian, E.R., Cohen, C.M.S., Cummings, A.C., Dwyer, J.R., Gold, R.E., Krimigis, S.M., Leske, R.A., Mewaldt, R.A., Slocum, P.L., Stone, E.C., von 
Rosenvinge, T.T.: 2002, Spectral properties of He and heavy ions in ${ }^{3} \mathrm{He}$-rich solar flares. Astrophys. $J$. 574, 1039 - 1058. doi:10.1086/341112.

Mason, G.M., Leske, R.A., Desai, M.I., Cohen, C.M.S., Dwyer, J.R., Mazur, J.E., Mewaldt, R.A., Gold, R.E., Krimigis, S.M.: 2008a, Abundances and energy spectra of corotating interaction region heavy ions observed during solar cycle 23. Astrophys. J. 678, 1458 - 1470. doi:10.1086/533524.

Mason, G.M., Korth, A., Walpole, P.H., Desai, M.I., von Rosenvinge, T.T., Shuman, S.A.: 2008b, The Suprathermal Ion Telescope (SIT) for the IMPACT/SEP investigation. Space Sci. Rev. 136, $257-284$. doi:10.1007/s11214-006-9087-9.

Mason, G.M., Desai, M.I., Mall, U., Korth, A., Bucik, R., von Rosenvinge, T.T., Simunac, K.D.: 2009, Insitu observations of CIRs on STEREO, Wind and ACE during 2007-2008. Solar Phys. 256, 393-408. doi:10.1007/s11207-009-9367-0.

Mazur, J.E., Mason, G.M., Klecker, B., McGuire, R.E.: 1993, The abundances of hydrogen, helium, oxygen, and iron accelerated in large solar particle events. Astrophys. J. 404, 810-817. doi:10.1086/172336.

Mazur, J.E., Mason, G.M., Dwyer, J.R., von Rosenvinge, T.T.: 1998, Solar energetic particles inside magnetic clouds observed with the Wind spacecraft. Geophys. Res. Lett. 25, 2521-2524. doi:10.1029/98GL00410.

Mewaldt, R.A., Cohen, C.M.S., Labrador, A.W., Leske, R.A., Mason, G.M., Desai, M.I., Looper, M.D., Mazur, J.E., Selesnick, R.S., Haggerty, D.K.: 2005, Proton, helium, and electron spectra during the large solar particle events of October-November 2003. J. Geophys. Res. 110, 9. doi:10.1029/2005JA011038.

Mewaldt, R.A., Cohen, C.M.S., Cook, W.R., Cummings, A.C., Davis, A.J., Geier, S., Kecman, B., Klemic, J., Labrador, A.W., Leske, R.A., Miyasaka, H., Nguyen, V., Ogliore, R.C., Stone, E.C., Radocinski, R.G., Wiedenbeck, M.E., Hawk, J., Shuman, S., von Rosenvinge, T.T., Wortman, K.: 2008a, The Low-Energy Telescope (LET) and SEP central electronics for the STEREO mission. Space Sci. Rev. 136, 285-362. doi:10.1007/s11214-007-9288-x.

Mewaldt, R.A., Cummings, A.C., Hu, Q., Leske, R., Acuna, M., Galvin, A., Gosling, J.T., Haggerty, D., Larson, D., Russell, C.: 2008b, STEREO and ACE observations of the May 2007 solar energetic particle events. In: 37th COSPAR Scientific Assembly 37, 2022.

Parker, E.N.: 1963, Interplanetary Dynamical Processes. Interscience, New York.

Reames, D.V.: 1990, Energetic particles from impulsive solar flares. Astrophys. J. Suppl. 73, 235 - 251. doi:10.1086/191456.

Reames, D.V.: 1995, Coronal abundances determined from energetic particles. Adv. Space Res. 15, $41-51$.

Reames, D.V., Barbier, L.M., Ng, C.K.: 1996, The spatial distribution of particles accelerated by coronal mass ejection - driven shocks. Astrophys. J. 466, 473 - 486. doi:10.1086/177525.

Richardson, I.G.: 1985, Low energy ions in co-rotating interaction regions at 1 AU: Evidence for statistical ion acceleration. Planet. Space Sci. 33, 557 - 569. doi:10.1016/0032-0633(85)90097-2.

Richardson, I.G.: 2004, Energetic particles and corotating interaction regions in the solar wind. Space Sci. Rev. 111, 267 - 376. doi:10.1023/B:SPAC.0000032689.52830.3e.

Richardson, I.G., Zwickl, R.D.: 1984, Low energy ions in corotating interaction regions at 1 AU: Observations. Planet. Space Sci. 32, 1179-1193. doi:10.1016/0032-0633(84)90143-0.

Richardson, I.G., Barbier, L.M., Reames, D.V., von Rosenvinge, T.T.: 1993, Corotating MeV/amu ion enhancements at $\leq 1 \mathrm{AU}$ from 1978 to 1986. J. Geophys. Res. 98, 13-32.

Roelof, E.C., Gold, R.E., Simnett, G.M., Tappin, S.J., Armstrong, T.P., Lanzerotti, L.J.: 1992, Low-energy solar electrons and ions observed at ULYSSES February-April, 1991 - The inner heliosphere as a particle reservoir. Geophys. Res. Lett. 19, $1243-1246$.

Sanderson, T.R., Bothmer, V., Marsden, R.G., Trattner, K.J., Wenzel, K.P., Balogh, A., Forsyth, R.J., Goldstein, B.E.: 1995, The Ulysses south polar pass: Energetic ion observations. Geophys. Res. Lett. 22, 3357 - 3360. doi:10.1029/95GL03089.

Scholer, M., Morfill, G., van Hollebeke, M.A.I.: 1980, On the origin of corotating energetic particle events. J. Geophys. Res. 85, $1743-1748$.

Schwadron, N.A., Fisk, L.A., Gloeckler, G.: 1996, Statistical acceleration of interstellar pick-up ions in corotating interaction regions. Geophys. Res. Lett. 23, 2871 - 2874. doi:10.1029/96GL02833.

Smith, E.J., Wolfe, J.H.: 1979, Fields and plasmas in the outer solar system. Space Sci. Rev. 23, 217 - 252. doi:10.1007/BF00173811.

Tan, L.C., Mason, G.M., Klecker, B., Hovestadt, D.: 1989, Seed population for about $1 \mathrm{MeV}$ per nucleon heavy ions accelerated by interplanetary shocks. Astrophys. J. 345, 572-583. doi:10.1086/167931.

Torsti, J., Anttila, A., Sahla, T.: 1999, Concurrent solar and corotating interaction region particle events in August 1996. J. Geophys. Res. 104, 9891 -9902. doi:10.1029/1998JA900170. 